\section{On the Use of Italic and Roman Fonts for Symbols in Scientific Text*}

\section{by lan Mills}

Scientific manuscripts frequently fail to follow the accepted conventions concerning the use of italic and roman fonts for symbols. An italic font is generally used for emphasis in running text, but it has a quite specific meaning when used for symbols in scientific text and equations. The following summary is intended to help in the correct use of italic fonts in preparing manuscript material.

1. The general rules concerning the use of italic (sloping) fonts or roman (upright) fonts are presented in Section 1.3.2., p. 5 and Section 4.1, p. 103 in the IUPAC Green Book [1], in relation to mathematical symbols and operators. These rules are also presented in the International Standards ISO 31 (successively being replaced by ISO/IEC 80000), ISO 1000 [2], and in the SI Brochure [3].

2. The overall rule is that symbols representing physical quantities or variables are italic, but symbols representing units, mathemathical constants, or labels are roman. Sometimes there may seem to be doubt as to whether a symbol represents a quantity or has some other meaning (such as a label): a good general rule is that quantities, or variables, may have a range of numerical values, but labels cannot. Vectors, tensors, and matrices are denoted using a boldface (heavy) font, but they should still be italic since they are still quantities.

\section{Examples:}

The mass of my pen $m=24 \mathrm{~g}=0.024 \mathrm{~kg}$. The electric field strength $E$ has components $E_{x}, E_{y}$, and $E_{z}$. The Planck constant $h=6.62606896(33) \times 10^{-34} \mathrm{~J} \mathrm{~s}$.

3. The above rule applies equally to letter symbols from both the Greek and the Latin alphabet, although authors often appear to resist putting Greek letters into italic.

\section{Example:}

When the symbol $\mu$ is used to denote a physical quantity (such as mass or reduced mass) it should be italic, but when it is used in a unit such as the microgram, $\mu \mathrm{g}$, or when it is used as the symbol for the muon, $\mu$ (see 5 below), it should be roman.

4. Numbers and labels are roman (upright), since they are not physical quantities.

Example:

The ground and first excited electronic states of the $\mathrm{CH}_{2}$ molecule are denoted

$\ldots\left(2 a_{1}\right)^{2}\left(1 b_{2}\right)^{2}\left(3 a_{1}\right)^{1}\left(1 b_{1}\right)^{1}, \widetilde{X}^{3} B_{1}$, and

$\ldots\left(2 a_{1}\right)^{2}\left(1 b_{2}\right)^{2}\left(3 a_{1}\right)^{2}, \tilde{a}^{1} A_{1}$, respectively.

The $\pi$-electron configuration and symmetry of the benzene molecule in its ground state are denoted: $\ldots\left(a_{2 u}\right)^{2}\left(e_{1 g}\right)^{4}, \widetilde{X}{ }^{1} A_{1 g}$. All these symbols are labels and are roman.

5. Symbols for elements in the periodic table should be roman, since they are not physical quantities. Similarly the symbols used to represent elementary particles are always roman. (See, however, paragraph 9 below for the use of italic font in chemical-compound names.)

\section{Examples:}

$\mathrm{H}, \mathrm{He}, \mathrm{Li}, \mathrm{Be}, \mathrm{B}, \mathrm{C}, \mathrm{N}, \mathrm{O}, \mathrm{F}, \mathrm{Ne}, \ldots$ for atoms; e for the electron, $\mathrm{p}$ for the proton, $\mathrm{n}$ for the neutron, $\mu$ for the muon, $\alpha$ for the alpha particle, etc.

6. Symbols for physical quantities are single letters of the Latin or Greek alphabet. Exceptionally two letters are used for certain dimensionless quantities, such as the Reynolds number, Re. However the symbols are frequently supplemented with subscripts or information in brackets to further specify the quantity. Further symbols used in this way are either italic or roman depending on whether they represent physical quantities or labels.

\section{Examples:}

$H$ denotes enthalpy, but $H_{\mathrm{m}}$ denotes molar enthalpy ( $\mathrm{m}$ is a mnemonic label for molar, and is therefore roman).

$C_{p}$ and $C_{V}$ denote the heat capacity at constant pressure $p$ and volume $V$, respectively; but $C_{p, m}$ and $C_{V, m}$ denote the molar heat capacity at constant $p$ and $V$, respectively (note the roman $m$ but italic $p$ and $V$ ).

The chemical potential of argon might be denoted $\mu_{\mathrm{Ar}}$ or $\mu(\mathrm{Ar})$, but the chemical potential of the ith component in a mixture would be denoted $\mu_{i}$, where the $i$ is italic because it is a variable index.

*The 1999 version of this document has been prepared by I.M. Mills and W.V. Metanomski and has since been available online. It was then slightly revised in 2007 and full text included in the GUIDELINES FOR DRAFTING IUPAC TECHNICAL REPORTS AND RECOMMENDATIONS and also in the 3rd edition of the IUPAC Green Book (ref. 1). 
7. Symbols for mathematical operators are always roman. This applies to the symbol $\Delta$ for a difference, $\delta$ for a small difference, $d$ for an infinitesimal difference (in calculus), and to capital $\Sigma$ and $\Pi$ for summation and product signs. The symbols $\pi$, e (base of natural logarithms), i (square root of minus one), etc. are always roman, as are the symbols for specified functions such as log (lg, In or lb), exp, sin, cos, tan, erf, div, grad, rot (the operators div, grad and rot may be printed boldface since they represent a vector). Some of these letters are also sometimes used to represent physical quantities: then of course they should be italic, to distinguish them from the corresponding mathematical symbol.

\section{Examples:}

$\Delta H=H($ final) $-H($ initial); $(\mathrm{d} p / \mathrm{d} t)$ used for the rate of change of pressure; $\delta x$ used to denote an infinitesimal variation of $x$. But for a damped linear oscillator the amplitude $F$ as a function of time $t$ might be expressed by the equation $F=F_{0} \exp (-\delta t) \sin (\omega t)$ where $\delta$ is the decay coefficient ( $\mathrm{SI}$ unit: $\mathrm{Np} / \mathrm{s}$ ) and $\omega$ is the angular frequency (SI unit: rad/s). Note the use of roman $\delta$ for the operator in a small time interval $\delta$, but italic $\delta$ for the decay coefficient in the product $\delta t$. Note that the products $\delta t$ and $\omega t$ are both dimensionless, but are described as having the unit neper $(\mathrm{Np}=1)$ and radian (rad $=1)$, respectively.

8. The fundamental physical constants are always regarded as quantities subject to measurement (even though they are not considered to be variables) and they should accordingly always be italic. Sometimes fundamental physical constants are used as though they were units, but they are still given italic symbols. An example is the hartree, $E_{h}$. However the electronvolt, eV, the dalton, Da, or the unified atomic mass unit, $u$, and the astronomical unit, ua, have been recognized as units by the Consultative Committee on Units of the BIPM and they are accordingly given roman symbols.

Examples:

$c_{0}$ for the speed of light in vacuum, $m_{\mathrm{e}}$ for the electron mass, $h$ for the Planck constant, $N_{\mathrm{A}}$ or $L$ for the Avogadro constant, e for the elementary charge, $a_{0}$ for the Bohr radius, etc. But for the electronvolt $\mathrm{eV}=\mathrm{e} \times \mathrm{V}$ $=1.602176487(40) \times 10^{-19} \mathrm{~J}$, the symbol eV is roman.
9. Greek letters are used in some cases for certain purposes in systematic organic, inorganic, polymer, and biochemical nomenclature. These should be in roman (upright) type. They designate e.g. the position of substituents, double bonds, ligating-atom attachment and bridging mode in coordination compounds, end groups in structure-based names for polymers, and configuration in carbohydrates and natural products. Letter symbols for elements are italic when used in names indicating attachments to heteroatoms, e.g. $O-, N-, S-$, and $P$-. The italic element symbol $H$ denotes indicated or added hydrogen [refs. 4 and 5].

\section{Examples:}

$\alpha$-ethylcyclopentaneacetic acid $\beta$-methyl-4-propylcyclohexaneethanol

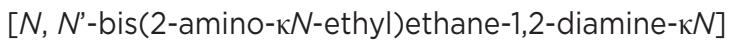
chloroplatinum(II)

tetracarbonyl $\left(\eta^{4}-2-\right.$ methylidenepropane-1,3-diyl) chromium

$\alpha$-(trichloromethyl)- $\omega$-chloropoly (1,4phenylenemethylene)

$\alpha$-D-glucopyranose

$5 \alpha$-androstan-3 $\beta$-ol

$\mathrm{N}$-methylbenzamide

O-ethyl hexanethioate

$3 \mathrm{H}$-pyrrole

naphthalen-2(1H)-one

\section{References}

1. Quantities, Units and Symbols in Physical Chemistry, the IUPAC Green Book, prepared for publication by E Richard Cohen, et al, 3rd Edition, Royal Society of Chemistry, Cambridge, 2007.

2. The ISO Standards Handbook, Quantities and Units, ISO, Geneva, 1993.

3. Le Système International d'Unités (the SI Brochure), 7th Edn. (French and English), BIPM, Sèvres, 1998.

4. Principles of Chemical Nomenclature,- A Guide to IUPAC Recommendations, 2011 Edition, edited by G J Leigh (RSC 2011, ISBN 978-1-84973-007-5).

5. Nomenclature of Organic Chemistry. IUPAC Recommendations and Preferred Names 2013, prepared by H A Favre and W H Powell (RSC 2013, ISBN 978-085404-182-4) 